Haben Sie Fragen zur Abrechnung oder zur wirtschaftlichen

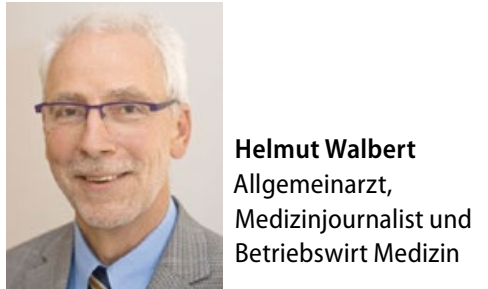

\title{
Sie können Ihre NäPA auf Impf-Tour schicken
}

1 Dr. P. W., Hausarzt, Bayern: In unserer großen hausärztlichen Landpraxis werden Impfungen in der Regel durch eine entsprechend qualifizierte Medizinische Fachangestellte (MFA) durchgeführt. Ist das auch bei Hausbesuchen ohne ärztliche Überwachung ohne Probleme möglich?

MMW-Experte Walbert: Jein! Es gibt KVen, die dies ablehnen, weil nur ein Arzt die Indikation stellen und die Impffähigkeit feststellen kann. Dies setzt also einen persönlichen Arzt-Patienten-Kon-

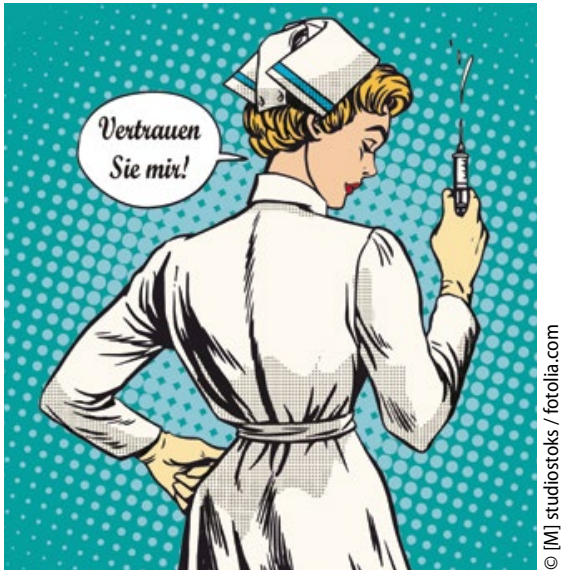

takt unmittelbar vor der Impfung voraus. Ist dies der Fall, kann die Nicht-ärztliche Praxisassistentin (NäPA) problemlos die Impfungen durchführen - auch im Rahmen von Hausbesuchen. Es empfiehlt sich, in diesen Fällen eine doppelte Dokumentation: einmal beim Arzt-PatientenKontakt („Impfindikation und Fähigkeit für Impfung festgestellt") und noch einmal bei Erbringung der Leistung durch die NäPA im Rahmen des Hausbesuchs („Arztkontakt lag vor“). Auf diese Weise sollte die Impfung rechts- und abrechnungssicher sein.

\section{Problemorientiertes Gespräch}

\section{GOP 03230 wird viel zu selten abgerechnet}

$?$ Dipl.-Med. H. V., Hausarzt, Thüringen: Wir haben immer wieder $\mathrm{Pa}$ tienten mit erheblichem Gesprächsbedarf. Wie oft kann im Behandlungsfall die Gesprächsleistung nach GOP 03230 abgerechnet werden? 03230 kann im Behandlungsfall unbegrenzt abgerechnet werden - so oft es eben $\mathrm{zu}$ einem „problemorientierten ärztlichen Gespräch aufgrund von Art und Schwere der Erkrankung" kommt. Der obligate Leistungsinhalt laut Legende ist ein Gespräch von mindestens zehn
Minuten Dauer mit dem Patienten und/ oder einer Bezugsperson.

Insgesamt ist die Abrechnung allerdings quotiert. Die GOP 03230 darf rechnerisch in 50\% der Behandlungsfälle angesetzt werden. Das ist normalerweise kein Problem, weil Patienten mit Akut- und Bagatellerkrankungen ja meist keinen Gesprächsbedarf haben. Deshalb ist der Blick auf die Uhr bei allen längeren Beratungen sinnvoll, und die GOP 03230 sollte stets angesetzt werden, wenn der Leistungsinhalt erbracht wurde. Viele Hausärzte rechnen die GOP viel zu selten $a b$ - manche sogar überhaupt nicht, wie aus der Leistungsstatistik zu ersehen ist.

Ein wichtiger Aspekt wird ebenfalls oft übersehen: Das Gespräch kann auch mit einer Bezugsperson geführt werden. Diese Situation tritt häufig in Altenheimen oder zu Hause bei geriatrischen Patienten ein. Auch bei entsprechenden Telefonaten kommt die Abrechnung infrage. Entscheidend ist der Inhalt des Gesprächs und die Dauer von mindestens zehn Minuten. Für die Plausibilitätsprüfung empfiehlt sich eine Kurzdokumentation. Die GOP geht in das Tages- und Quartalszeitprofil ein. 